\title{
Inventaire de l'entomofaune de cocoteraies infectées par la maladie du jaunissement mortel du cocotier de trois villages du département de Grand- Lahou (Adjadon, Badadon et Yaokro) en Côte d'Ivoire
}

\author{
N'Djiha Isabelle BEUGRE ${ }^{1,2 *}$, Koffi Eric KWADJO ${ }^{1}$, Kouadio Dagobert KRA ${ }^{1}$, \\ Aubin Silvère Djiwha DANON ${ }^{1}$, Kouamé Kan Sébastien LOUKOU ${ }^{1}$, \\ Weintoin Alimata Marie Pierre DARAMCOM ${ }^{2}$, Kouassi ALLOU $^{2}$ et \\ Dagnogo MAMADOU ${ }^{1}$
}

${ }^{1}$ UFR Sciences de la Nature, Laboratoire de Biologie et Cytologie Animales, Université Nangui Abrogoua, 02 BP 801 Abidjan 02, Côte d'Ivoire.

${ }^{2}$ Centre National de Recherche Agronomique, Station de Recherche Marc DELORME, Laboratoire de Défense des cultures sur le programme cocotier, 07 BP 13 Abidjan 07, Côte d'Ivoire.

*Auteur correspondant ; E-mail: isabelle.bn1@gmail.com,Tél.: +225 59491813

\section{RESUME}

Le cocotier, Cocos nucifera L. (Arecaceae) est une oléagineuse pérenne, cultivé dans les régions côtières de la zone intertropicale de la Côte d'Ivoire. Cependant, la plante est sujette à des dégâts causés par les insectes. Ce travail vise à connaître les différents ordres et familles d'insectes vivant dans les cocoteraies infectées par la maladie du jaunissement mortel du cocotier dans le département de Grand-Lahou, Côte d'Ivoire. Les échantillons d'insectes ont été collectés dans trois villages du département de Grand-Lahou à savoir Adjadon, Badadon et Yaokro. Deux types de pièges dont les pièges jaunes et les pièges à fosse ont été utilisés dans cette étude. Ainsi, 15171,7743 et 16600 insectes ont été collectés respectivement à Adjadon, à Badadon et à Yaokro. L'occurrence des insectes déprédateurs sur les différentes cocoteraies est la même pour les trois sites. Ces insectes déprédateurs ont été attaqués par des insectes prédateurs et parasitoïdes qui contribuent ainsi à la régulation naturelle de leur population. En outre, les insectes ont été moins diversifiés sur les différents sites d'étude avec des indices de diversité de Shannon inférieurs à 1,5 bits et des indices d'équitabilité inférieurs à 50\%. Cette étude implique une actualisation de la liste des insectes du cocotier face aux différents dégâts observés dans les différentes zones de culture.

(C) 2018 International Formulae Group. All rights reserved.

Mots clés : Cocoteraie, Côte d'Ivoire, déprédateurs, parasitoïdes.

\section{Entomofauna inventory of the coconut palm sites infected by coconut lethal yellowing in the Grand-Lahou department (Adjadon, Badadon and Yaoko), Côte d'Ivoire}

\begin{abstract}
The coconut palm, Cocos nucifera L. (Arecaceae) is a perennial oil plant, grown in the coastal regions of the intertropical zone of Côte d'Ivoire. However, the plant is subject to damages caused by the insects. This study aimed at determining the orders and families of the insects living in the coconut palm sites
\end{abstract}


infected by coconut lethal yellowing in the Grand-Lahou division, Côte d'Ivoire. The insect samples were collected in three villages of Grand-Lahou division, including Adjadon, Badadon and Yaokro. Two types of insect traps, such as pitfall trap and yellow trap were used in the study. A total of 15171,7743 and 16600 insects were collected respectively in Adjadon, in Badadon and in Yaokro. Occurrence of the depredator insect on various coconut palm sites is the same for the three sites. These depredator insects were attacked by predators and parasitoids insects which contribute to the natural regulation of the depredators populations. Besides, the insects were diversified less on the different coconut palm sites with diversity indices of Shannon $<1,5$ bits and equitabilities $<50 \%$. This study implies actualization of the list of coconut insects towards different damages observed in different coconut farming areas.

(C) 2018 International Formulae Group. All rights reserved.

Keywords: Coconut plantation, Côte d'Ivoire, depredators, parasitoïds

\section{INTRODUCTION}

Le cocotier Cocos nucifera L. (Arecaceae) est une plante pérenne, oléagineuse et sucrière originaire des bassins Indo-Atlantique et Pacifique (Gunn et al., 2011). La nuciculture est pratiquée dans les régions côtières de la zone intertropicale. Le cocotier est souvent appelé "Arbre de vie" par certains auteurs car tous ses organes sont utiles à l'homme. Il est utilisé dans différents domaines, notamment l'alimentation, le cosmétique, la pharmacopée et l'artisanat (Van der Vossen et Chipungahelo, 2007). La surface occupée par les cocoteraies dans le monde, est estimée à plus de 12 millions hectares pour une production annuelle de 5, 2 à 5,8 millions de tonnes de coprah selon FAO et Oil World (2010). En Côte d'Ivoire, la culture du cocotier se réalise sur une superficie d'environ 50000 ha pour une production annuelle de plus de $70000 \mathrm{t}$ de coprah (CNRA, 2014). D'après les statistiques fournies par le CNRA (2006), les plantations villageoises et industrielles occupent respectivement $60 \%$ et $40 \%$ des surfaces cultivées de cocotier en Côte d'Ivoire. Le cocotier est essentiellement cultivé sur le littoral ivoirien et fait vivre plus de 20000 familles (Assa et al., 2006). Il représente, par conséquent, pour les producteurs et les utilisateurs, un enjeu à la fois économique, social et culturel.
Toutefois, l'enjeu économique que représente cette plante au plan mondial, est compromis par plusieurs contraintes parmi lesquels le vieillissement des cocoteraies, la faiblesse de la production, l'incidence des ravageurs et des maladies (Allou, 2009).

En Côte d'Ivoire, les plus graves maladies observées étaient dues au champignon Phytophthora katsurae. Mais depuis 1995, dans le département de GrandLahou, ville située à une centaine de kilomètres d'Abidjan et grande productrice de noix de coco, une autre maladie, très dévastatrice a été observée dans différentes plantations (Amenan et al., 2012). Elle détruit d'importantes surfaces de cocoteraie. Selon les premières estimations faites par le CNRA en 2012, près de $70 \%$ des cocotiers du département de Grand-Lahou sont détruits par ce fléau (CNRA, 2012). Cette maladie pourrait également menacer l'une des plus importante collection ex situ de cocotier du réseau international Coconut Genetic Network (COGENT), la collection internationale pour l'Afrique et l'Océan Indien située à Abidjan $\mathrm{du}$ fait des migrations importantes des populations (Konan et al., 2013).

Une enquête épidémiologique conduite par le Centre National de Recherche Agronomique (CNRA) et financée par le Fonds Interprofessionnel pour la Recherche et le Conseil Agricoles (FIRCA) a permis de déterminer la cause de cette mortalité des 
cocotiers, d'évaluer la situation sanitaire générale du verger cocotier, de recenser les plantations affectées puis d'évaluer l'intensité de l'infection dans 6 zones du département de Grand-Lahou. L'analyse des échantillons d'organes symptomatiques de cocotier a permis de conclure en 2013 que la mortalité des cocotiers de Grand-Lahou est causée par un phytoplasme baptisé Côte d'Ivoire Lethal Yellowing phytoplasma ou CILYP (Konan et al., 2013). Ainsi, ce pathogène est à l'origine de la maladie du Jaunissement Mortel du Cocotier (JMC) couramment appelée par les paysans « La maladie des cocotiers de GrandLahou » (CNRA, 2013). La maladie du jaunissement mortel du cocotier demeure donc actuellement le risque phytosanitaire le plus inquiétant auquel est confronté la nuciculture dans le monde. En dépit des programmes internationaux de recherche (Caraïbes, Floride, etc.) il n'existe pas encore de remède (Cirad, 2014). Les méthodes de lutte actuellement utilisées sont physiques ou génétiques.

Sur le littoral de la Côte d'Ivoire, les villes de Jacqueville et Grand Lahou possèdent de grands vergers de cocotier (Assa et al., 2006). Cependant à Grand Lahou, cette maladie s'est rapidement développée dans les cocoteraies affectant actuellement plus de 7 000 hectares (ProMED, 2013). Ainsi, plus de 100 familles de planteurs de cocotier ont perdu leur revenu induisant l'augmentation de la pauvreté dans la région. Toute la cocoteraie ivoirienne est menacée par la propagation de la maladie d'autant plus qu'à ce jour, il n'existe aucune méthode de lutte chimique. C'est donc pour cerner les contours de ce fléau en vue de mieux le contrôler et réduire son impact socio-économique que le Centre National de Recherche Agronomique (CNRA), à travers son Programme Cocotier, conduit des travaux de recherche visant à faire l'inventaire de l'entomofaune de la cocoteraie infectée de la maladie du jaunissement mortel $\mathrm{du}$ cocotier de trois villages dans le département de Grand-Lahou.

Le présent travail est en faveur d'une identification des différents ordres et familles d'insectes vivant dans les cocoteraies infectées par la maladie du jaunissement mortel du cocotier de Grand-Lahou.

\section{MATERIEL ET METHODES Zone d'étude}

La collecte des insectes a été réalisée dans des cocoteraies infectées par la maladie $\mathrm{du}$ jaunissement mortel du cocotier de trois localités du département de Grand-Lahou, située au sud de la Côte d'Ivoire, dans la région des lagunes entre $5^{\circ} 144^{\prime \prime}$ de latitude nord et $5^{\circ} 00^{\prime} 11^{\prime \prime}$ de longitude ouest, au bord du golfe de Guinée, à l'embouchure du fleuve Bandama. Elle présente un paysage lagunaire et maritime à côte sableuse et est caractérisée par des sols ferralitiques faiblement et/ou fortement dénaturés.

Les captures d'insectes se sont déroulées dans trois localités à savoir Adjadon, Badadon et Yaokro, situées sur une île (entre l'océan et la lagune). Les sites d'Adjadon et de Badadon sont orientés vers l'océan avec des sols beaucoup sableux et le site de Yaokro orienté vers le continent avec un sol qui a les caractéristiques de celui du continent (moins sableux). Les 3 parcelles sur lesquelles ont été posés les pièges avaient une superficie allant de 1 à 3 ha. La pluviométrie enregistrée pendant la période d'étude allant d'octobre 2015 à septembre 2016 a été plus élevée en octobre 2015 (318 mm) qu'en février $2016(11,5 \mathrm{~mm})$. La température moyenne a été de $27,5^{\circ} \mathrm{C}$ (Figure 1).

\section{Matériel de piégeage des insectes}

La collecte des insectes inféodés à la cocoteraie infectée de Grand-Lahou a été réalisée à l'aide de pièges jaunes constitués de récipients de couleurs jaunes remplis aux $1 / 4$ d'eau additionnée de mouillant (Figure 2) et 
de pièges à fosse constitués de gobelets plastiques (Figure 3).

\section{Collecte des insectes}

La capture des insectes avec ces différents pièges a été réalisée trois fois par mois. Les pièges jaunes ont servi à capturer les insectes volants et attirés par la couleur. Ces pièges ont été posés au niveau de la strate herbacée. Les pièges à fosse, remplis aux $1 / 3$ d'eau salée additionnée de mouillant et placés dans des trous préalablement creusés dans le sol, ont servi à capturer les insectes se déplaçant au niveau du sol. Ces deux type de pièges ont été disposés sur deux transects de cinquante mètres chacun et distants l'un de l'autre de cinq mètres. Sur chaque transect, cinq pièges à fosse et cinq pièges jaunes alternés et distants les uns des autres de cinq mètres ont été disposés. Au total, 20 pièges ont été utilisés dont 10 pièges jaunes et 10 pièges à fosses.

\section{Conservation et identification des spécimens d'insectes}

Les insectes capturés par les pièges ont été conservés dans des piluliers contenant de l'alcool à $70 \%$ après le passage du contenu des récipients à travers un tamis de mailles 0,5 $\mathrm{mm}$. Des étiquettes portant la date, le nom du site et le type de piège ont été apposées sur le couvercle des piluliers contenant les insectes puis ces piluliers ont été acheminés au laboratoire pour y effectuer le dénombrement et l'identification des niveaux taxonomiques que sont l'ordre et la famille. La clé de reconnaissance de Delvare et Aberleng (1989), la clé d'identification des collections de Roth (1980) ainsi que la clé dichotomique d'identification ont été utilisés pour la reconnaissance des unités taxonomiques dans lesquelles les insectes collectés ont été classifiés.

\section{Analyses statistiques}

La constance et la diversité des ordres et familles d'insectes ont été calculées. La constance (C) exprimée en pourcentage (Dajoz, 2006) est le rapport entre le nombre de relevés contenant la famille étudiée (Pi) et le nombre total de relevés $(\mathrm{P})$. La formule générale est la suivante :

$C(\%)=\frac{P i}{P} \times 100$

Où $\mathrm{P}_{\mathrm{i}}$ : nombre de relevés contenant l'ordre, la famille ou l'espèce étudié ; $\mathrm{P}$ : nombre total des relevés effectués. Suivant les variations de la valeur de $\mathrm{C}$, une catégorisation a été effectuée au sein de l'unité taxonomique étudiée selon Bigot et Bodot (1973) : C $\geq$ $50 \%$ : les familles sont qualifiées de constantes ; $25 \% \leq \mathrm{C} \leq 49 \%$ : les familles sont qualifiées d'accessoires ; $10 \% \leq \mathrm{C} \leq 25 \%$ : les familles sont qualifiées d'accidentelles et $\mathrm{C} \leq 10 \%$ : les familles très accidentelles, qualifiées de sporadiques.

Pour évaluer la diversité des ordres d'insectes, l'indice de diversité de Shannon qui fait appel à la théorie de l'information a été choisi. Cet indice tient compte du fait que la diversité est fonction de la probabilité (pi) de présence de chaque espèce ou de chaque taxon dans un groupe d'individus (Dajoz, 1982). Ainsi, le calcul de l'indice de diversité de Shannon doit toujours s'accompagner de celui de l'équitabilité (E). La formule générale de l'indice de diversité de Shannon (H) est donnée par :

$\mathrm{H}=-\mathrm{S}$ pi $\log 2 \mathrm{pi}$

On définit l'équitabilité (E), comme le rapport $\mathrm{H} / \mathrm{Hmax}$

$\mathrm{H} \max =\log 2 \mathrm{n}$ où $\mathrm{n}$ correspond à la valeur maximale de $\mathrm{H}$ lorsque toutes les familles ont la même abondance. L'équitabilité (E) varie de 0 à 1 . 


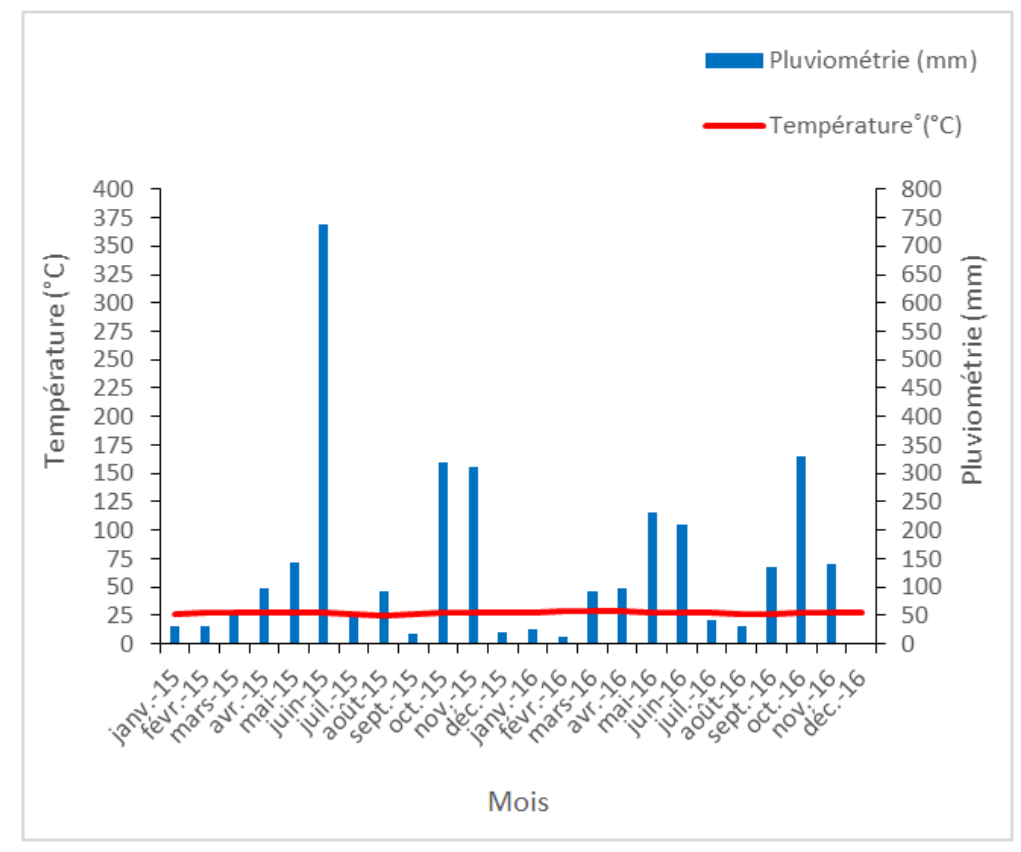

Figure 1. Précipitations et températures moyennes recensées au cours de l'étude à Grand-Lahou (Station de TOUPAH) de 2015 à 2016.

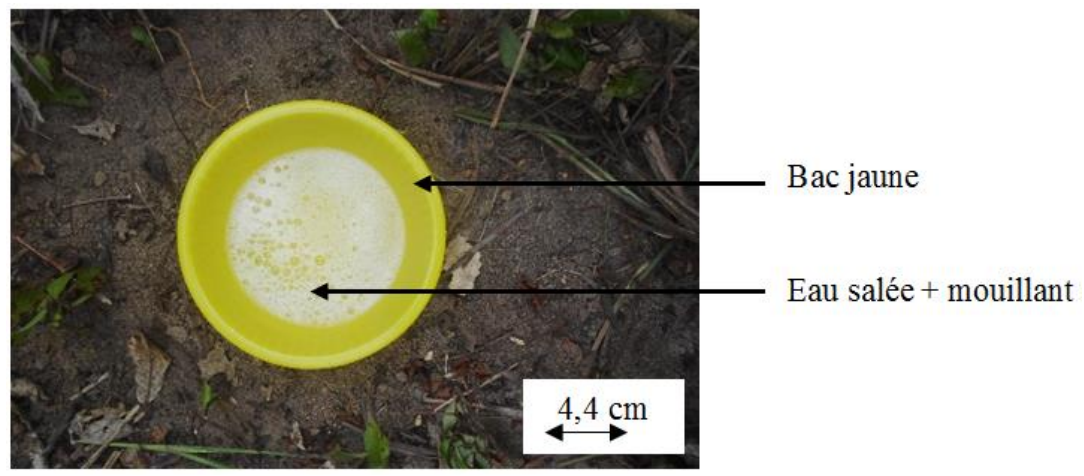

Figure 2 : Piège à bac jaune.

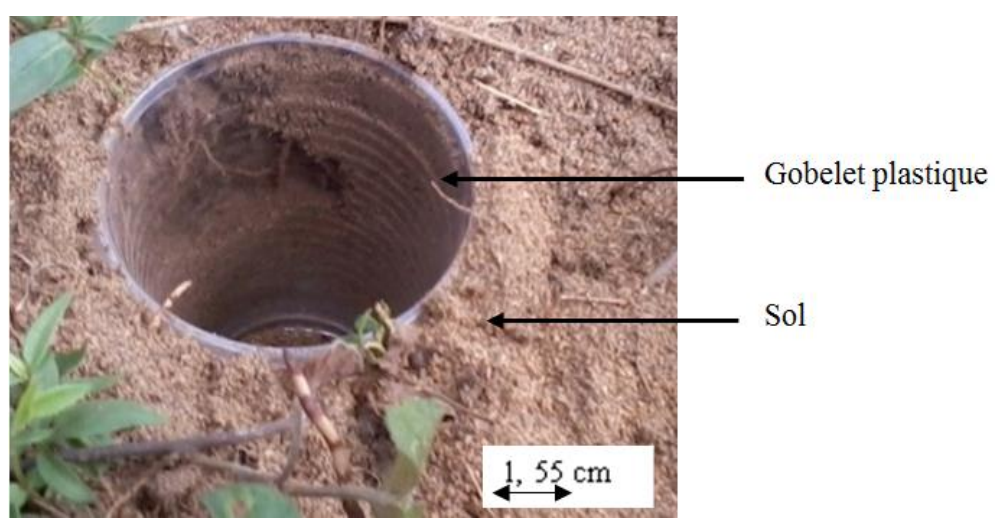

Figure 3 : Piège à fosse. 


\section{RESULTATS \\ Répartition globale des insectes sur les sites d'étude}

Au total, 39514 insectes, repartis en 15 ordres et 134 familles, ont été récoltés. Le Tableau 1 montre qu'à Adjadon, 15171 insectes regroupés en 12 ordres et 111 familles ont été récoltés dont les plus importants sont les Diptères (31 familles), Hyménoptères (23 familles) et les Coléoptères (12 familles). A Badadon, la récolte a révélé la présence de 7743 insectes repartis en 14 ordres et 96 familles (Tableau 1). Les Diptères, Hyménoptères et Coléoptères avec respectivement 27, 20 et 18 familles sont les plus nombreux. Sur le site de Yoakro, 16600 insectes, repartis en 14 ordres et 107 familles, ont été collectés (Tableau 1). Les plus représentés sont les Diptères, les Hyménoptères et les Coléoptères, avec respectivement 27,22 et 21 familles.

\section{Répartition des insectes ravageurs de cocotier sur les sites d'étude}

A Adjadon, les insectes ravageurs récoltés dans les cocoteraies appartenaient à 4 ordres et 4 familles repartis en constantes, accessoires, accidentelles et très accidentelles. Les familles constantes étaient les Chrysomelidae, Cicadellidae, Acrididae et Gryllidae, avec des constantes comprises entre 69,44 et $97,22 \%$ pour des pourcentages de 0,79 à $4,94 \%$. Les accessoires regroupaient 4 familles, avec des constances allant de 25 à $44,44 \%$ et des pourcentages dans le peuplement total de 0,11 à $1,01 \%$. Les familles accidentelles ont appartenu à 4 ordres, avec des constances de 11,11 à $22,22 \%$ et des pourcentages dans le peuplement total de 0,02 à $0,17 \%$. Les familles très accidentelles ont concerné les Delphacidae. Elles ont eu une constance de $2,77 \%$ pour un pourcentage de $0,006 \%$ dans le peuplement total (Tableau 2). A Badadon, les insectes ravageurs récoltés ont concerné 5 ordres et 12 familles réparties comme suit : Des familles constantes ont appartenu à 3 ordres, avec des constances comprises entre 63,88 et $97,22 \%$ pour des pourcentages de 0,39 à $4,33 \%$. Les accessoires ont concerné 4 familles, avec des constances allant de 25 à $47,22 \%$ pour des pourcentages qui ont varié entre 0,11 et $0,63 \%$ du peuplement total. Les familles accidentelles ont appartenu à 2 ordres, ceux des Homoptères et des Orthoptères. Leurs constances ont été de 16,66 à $11,11 \%$ pour des pourcentages de 0,09 et $0,17 \%$. Les familles très accidentelles ont concerné 2 familles, avec une constance de $5,55 \%$ chacune et des pourcentages dans le peuplement total de 0,02 et $0,01 \%$ (Tableau 2). Sur la parcelle de Yaokro, les insectes ravageurs récoltés ont été regroupés en 5 ordres et 13 familles (Tableau 2) qui ont appartenu à des familles constantes, accessoires, accidentelles et très accidentelles. Les familles constantes ont concerné 5 familles. Leurs constances ont varié entre 69,44 et $100 \%$ et des pourcentages de 0,53 à $10,38 \%$. Les familles accessoires ont elles appartenu à 3 ordres, avec des constances comprises entre 33,33 et $36,11 \%$ pour des pourcentages de 0,13 à $1,33 \%$ du peuplement total. Quant aux familles accidentelles, elles ont appartenu aux Hesperiidae avec une constance de $19,44 \%$ et un pourcentage de $0,07 \%$. Les très accidentelles ont appartenu à 4. Leurs constances ont varié entre 2,77 et $8,33 \%$ pour des pourcentages comprises entre 0,006 et $0,05 \%$ du peuplement total (Tableau 2).

\section{Répartition des insectes prédateurs et parasitoïdes sur les sites d'étude}

Les insectes prédateurs et parasitoïdes récoltés à Adjadon ont appartenu à 7 ordres repartis en 33 familles (Tableau 3). Les familles constantes ont eu des constances qui ont varié de 52,77 à $100 \%$. Ces familles ont eu des pourcentages dans le peuplement total de 0,30 et $41,89 \%$. Les familles accessoires sont constituées de 5 familles. Leurs constances ont été de 25 à $44,44 \%$ pour des pourcentages de 0,09 à 0,59 dans le peuplement total. Les familles accidentelles, ont appartenu à 3 ordres avec des constances qui ont varié de 11,11 à $22,22 \%$. Leurs pourcentages dans le peuplement total ont été de 0,01 à $0,17 \%$. Les très accidentelles avec des constances de 2,77 à $8,33 \%$, ont concerné 10 familles. Ces 
familles ont enregistré des populations très faibles de 0,006 et $0,05 \%$. A Badadon, les prédateurs et parasitoïdes capturés ont appartenu à 5 ordres repartis en 8 familles (Tableau 3). Les familles constantes avec des constances de 55,55 à 100\%, ont présenté une partie des populations avec un taux de 0,86 à $25,54 \%$. Les familles accessoires avec des constances de 25 à $47,22 \%$ ont appartenu à 4 ordres. Ces ordres ont enregistré des pourcentages de 0,24 à $1,12 \%$. Les familles accidentelles ont Concerné 6 familles avec des constances de 11,11 à $22,22 \%$. Elles ont des populations faibles comprises entre 0,11 et $0,38 \%$. Les familles très accidentelles ont appartenu à 7 ordres. Ces ordres ont eu des constances respectives de 2,77 et 5,55\% et des populations très faibles avec des pourcentages de 0,01 et $0,14 \%$. Quant à Yaokro, les insectes prédateurs et parasitoïdes récoltés ont appartenu à 8 ordres et repartis en 34 familles parmi lesquels, 9 constantes, 5 accessoires, 7 accidentelles et 13 très accidentelles (Tableau 3). Les familles constantes ont eu des constances de 50 à $100 \%$ avec des pourcentages de 0,24 à $44,56 \%$ du peuplement total. Les familles accessoires sont constituées de 5 familles. Leurs constances ont varié de 27,77 à $44,44 \%$. Elles ont présenté des taux allant de 0,18 à $0,61 \%$ du peuplement total. Les familles accidentelles, ont été regroupées en 5 ordres et 7 familles avec des constances de 11,11 à $16,66 \%$. Ceux-ci ont des populations dont les pourcentages sont de 0,03 et $0,12 \%$. Quant aux autres familles, elles sont donc dites très accidentelles. Leurs constances sont de 2,77 à $8,33 \%$, avec des populations très faibles de 0,006 à $0,16 \%$.

\section{Diversité des ordres d'insectes sur les sites d'étude}

A Adjadon, tous les ordres d'insectes obtenus ont présenté des indices de diversité faibles allant de 0,132 à 1,942 bits (Tableau 4). La moyenne des indices de diversité de Shannon calculé pour ce site a été de 0,81 bits avec une équitabilité de 0,411 . Par contre à Badadon, l'ordre des Diptères a enregistré un indice de diversité de 2,6 bits tandis que les autres ordres ont présenté des indices faibles de 0,356 à 1,82 bits (Tableau 4). La moyenne des indices de diversité de Shannon calculés a été de 0,773 bits avec une équitabilité de 0,429. Sur la parcelle de Yaokro également, seuls les Diptères ont observé un indice de diversité de 2,453 bits tandis que les autres ont obtenu des indices faibles de 0,165 à 1,708 bits (Tableau 4). La moyenne des indices de diversité de Shannon de cette parcelle a été de 0,708 bits et une équitabilité équivalente à 0,414 .

Tableau 1: Inventaire des insectes collectés sur des sites à Grand-Lahou.

\begin{tabular}{llccc}
\hline \multirow{2}{*}{ Ordres } & & \multicolumn{3}{c}{ Effectif d'insectes } \\
\cline { 3 - 5 } & Familles & Adjadon & Badadon & Yaokro \\
\hline Coléoptères & Alticidae & 2 & 0 & 0 \\
& Anobiidae & 2 & 0 & 0 \\
& Anthicidae & 10 & 5 & 6 \\
& Apionidae & 6 & 5 & 3 \\
& Bostrichidae & 3 & 3 & 8 \\
& Buprestidae & 0 & 0 & 0 \\
& Bruchidae & 9 & 0 & 59 \\
& Carabidae & 25 & 43 & 1 \\
& Cerambycidae & 2 & 4 & 81 \\
& Chrysomelidae & 215 & 56 & 1
\end{tabular}




\begin{tabular}{|c|c|c|c|c|}
\hline & Coccinellidae & 871 & 43 & 48 \\
\hline & Curculionidae & 9 & 4 & 7 \\
\hline & Elateridae & 6 & 5 & 10 \\
\hline & Geotrupidae & 0 & 0 & 6 \\
\hline & Histeridae & 95 & 51 & 122 \\
\hline & Latridiidae & 2 & 0 & 0 \\
\hline & Nitidulidae & 7 & 13 & 30 \\
\hline & Rutelidae & 6 & 9 & 7 \\
\hline & Scarabaeidae & 154 & 96 & 528 \\
\hline & Silvanidae & 0 & 2 & 1 \\
\hline & Staphylinidae & 7 & 15 & 19 \\
\hline & Tenebrionidae & 16 & 13 & 20 \\
\hline & Trogidae & 2 & 0 & 1 \\
\hline Collemboles & Isotomidae & 49 & 260 & 2154 \\
\hline Dermaptères & Forficulidae & 0 & 1 & 0 \\
\hline \multirow[t]{2}{*}{ Dictyoptères } & Blattidae & 66 & 54 & 123 \\
\hline & Mantidae & 2 & 7 & 5 \\
\hline \multirow[t]{32}{*}{ Diptères } & Acroceridae & 1 & 0 & 0 \\
\hline & Agromyzidae & 13 & 43 & 29 \\
\hline & Anthomyiidae & 9 & 0 & 0 \\
\hline & Asilidae & 10 & 9 & 6 \\
\hline & Bibionidae & 1 & 1 & 1 \\
\hline & Calliphoridae & 134 & 222 & 118 \\
\hline & Chironomidae & 15 & 34 & 48 \\
\hline & Chloropidae & 6 & 4 & 5 \\
\hline & Conopidae & 76 & 4 & 3 \\
\hline & Diopsidae & 6 & 19 & 27 \\
\hline & Dolichopodidae & 91 & 59 & 58 \\
\hline & Drosophilidae & 588 & 144 & 142 \\
\hline & Empididae & 1 & 0 & 0 \\
\hline & Lonchaeidae & 5 & 3 & 6 \\
\hline & Lonchopteridae & 1 & 1 & 2 \\
\hline & Micropezidae & 93 & 53 & 135 \\
\hline & Muscidae & 78 & 41 & 109 \\
\hline & Mycetophilidae & 1 & 4 & 1 \\
\hline & Phoridae & 337 & 153 & 243 \\
\hline & Pipunculidae & 4 & 0 & 1 \\
\hline & Psychodidae & 7 & 3 & 4 \\
\hline & Sarcophagidae & 619 & 20 & 202 \\
\hline & Scatopsidae & 1 & 0 & 0 \\
\hline & Sciaridae & 18 & 25 & 9 \\
\hline & Sciomyzidae & 1 & 0 & 3 \\
\hline & Sepsidae & 0 & 21 & 0 \\
\hline & Simuliidae & 3 & 7 & 9 \\
\hline & Stratiomyidae & 67 & 77 & 89 \\
\hline & Syrphidae & 19 & 67 & 41 \\
\hline & Tabanidae & 0 & 5 & 0 \\
\hline & Tachnidae & 2 & 0 & 0 \\
\hline & Tephritidae & 2 & 3 & 4 \\
\hline
\end{tabular}




\begin{tabular}{|c|c|c|c|c|}
\hline & Therevidae & 0 & 5 & 0 \\
\hline & Tipulidae & 0 & 0 & 3 \\
\hline & Trypetidae & 1402 & 124 & 367 \\
\hline Embioptères & Oligotomidae & 0 & 0 & 1 \\
\hline \multirow{7}{*}{ Hétéroptères } & Coreidae & 4 & 0 & 0 \\
\hline & Cydnidae & 0 & 0 & 1 \\
\hline & Lygaeidae & 0 & 0 & 1 \\
\hline & Miridae & 0 & 2 & 12 \\
\hline & Pyrrhocoridae & 11 & 15 & 22 \\
\hline & Reduviidae & 47 & 56 & 10 \\
\hline & Tingidae & 2 & 0 & 0 \\
\hline \multirow[t]{11}{*}{ Homoptères } & Aphididae & 356 & 764 & 130 \\
\hline & Cercopidae & 12 & 23 & 22 \\
\hline & Cicadellidae & 506 & 268 & 512 \\
\hline & Cicadidae & 3 & 2 & 3 \\
\hline & Delphacidae & 1 & 0 & 1 \\
\hline & Derbidae & 26 & 14 & 20 \\
\hline & Dictyopharidae & 20 & 20 & 28 \\
\hline & Flatidae & 1 & 14 & 2 \\
\hline & Fulgoridae & 5 & 0 & 5 \\
\hline & Membracidae & 79 & 44 & 15 \\
\hline & Psyllidae & 59 & 53 & 28 \\
\hline \multirow[t]{27}{*}{ Hyménoptères } & Anthophoridae & 80 & 28 & 48 \\
\hline & Apidae & 17 & 24 & 7 \\
\hline & Bethylidae & 249 & 70 & 126 \\
\hline & Braconidae & 0 & 2 & 20 \\
\hline & Cephidae & 6 & 0 & 8 \\
\hline & Ceraphronidae & 2 & 0 & 0 \\
\hline & Chalcididae & 165 & 87 & 106 \\
\hline & Crabronidae & 18 & 1 & 1 \\
\hline & Diapriidae & 0 & 0 & 3 \\
\hline & Elasmidae & 0 & 0 & 1 \\
\hline & Encyrtidae & 14 & 20 & 14 \\
\hline & Eucharidae & 0 & 0 & 2 \\
\hline & Evaniidae & 18 & 19 & 30 \\
\hline & Figitidae & 4 & 0 & 0 \\
\hline & Formicidae & 6356 & 1978 & 7397 \\
\hline & Halictidae & 113 & 53 & 41 \\
\hline & Ibaliidae & 3 & 0 & 0 \\
\hline & Ichneumonidae & 81 & 29 & 61 \\
\hline & Megachilidae & 1 & 1 & 0 \\
\hline & Mutillidae & 4 & 0 & 0 \\
\hline & Platygastridae & 0 & 11 & 27 \\
\hline & Pteromalidae & 20 & 30 & 42 \\
\hline & Scelionidae & 158 & 120 & 296 \\
\hline & Scoliidae & 27 & 3 & 0 \\
\hline & Sphecidae & 8 & 19 & 8 \\
\hline & Stephanidae & 148 & 43 & 52 \\
\hline & Tenthredinidae & 4 & 4 & 0 \\
\hline
\end{tabular}




\begin{tabular}{|c|c|c|c|c|}
\hline & Trichogrammatidae & 0 & 0 & 5 \\
\hline & Vespidae & 149 & 153 & 243 \\
\hline Isoptères & Termitidae & 82 & 70 & 203 \\
\hline \multirow[t]{10}{*}{ Lépidoptères } & Cossidae & 0 & 2 & 3 \\
\hline & Hesperiidae & 18 & 20 & 12 \\
\hline & Lycaenidae & 1 & 0 & 0 \\
\hline & Noctuidae & 20 & 18 & 25 \\
\hline & Nymphalidae & 4 & 5 & 4 \\
\hline & Papillionidae & 6 & 0 & 0 \\
\hline & Pieridae & 4 & 0 & 0 \\
\hline & Pyralidae & 2 & 0 & 0 \\
\hline & Saturniidae & 36 & 8 & 41 \\
\hline & Tineidae & 1 & 0 & 0 \\
\hline \multirow[t]{3}{*}{ Neuroptères } & Coniopterygidae & 0 & 0 & 3 \\
\hline & Mantispidae & 0 & 1 & 2 \\
\hline & Nemopteridae & 0 & 2 & 2 \\
\hline \multirow[t]{2}{*}{ Odonates } & Coenagriidae & 1 & 3 & 2 \\
\hline & Lestidae & 1 & 0 & 1 \\
\hline \multirow[t]{5}{*}{ Orthoptères } & Acrididae & 121 & 166 & 176 \\
\hline & Gryllidae & 750 & 658 & 1575 \\
\hline & Pyrgomorphidae & 50 & 61 & 9 \\
\hline & Tetrigidae & 68 & 95 & 149 \\
\hline & Tettigoniidae & 17 & 26 & 9 \\
\hline Thysanoptères & Thripidae & 9 & 5 & 9 \\
\hline Total & & 15171 & 7743 & 16600 \\
\hline
\end{tabular}

Tableau 2 : Ordres, familles, nombre d'insectes, taux de représentativité des ravageurs et constances des familles d'insectes ravageurs de cocotier recensés dans les sites d'étude à GrandLahou.

\begin{tabular}{llccc}
\hline Sites d'étude & Ordres / familles & $\begin{array}{c}\text { Nombre } \\
\text { d'insectes }\end{array}$ & $\begin{array}{c}\text { Taux de représentativité } \\
\text { des ravaeurs (\%) }\end{array}$ & $\begin{array}{c}\text { Constance } \\
(\boldsymbol{\%})\end{array}$ \\
\hline Adjadon & Coléoptères / Chrysomelidae & 215 & 1,41 & 80,55 \\
& Coléoptères / Curculionidae & 9 & 0,05 & 19,44 \\
& Coléoptères / Scarabaeidae & 154 & 1,01 & 44,44 \\
& Hétéroptères / Coreidae & 4 & 0,02 & 11,11 \\
& Homoptères / Cicadellidae & 506 & 3,33 & 86,11 \\
& Homoptères / Delphacidae & 1 & 0,006 & 2,77 \\
& Homoptères / Derbidae & 26 & 0,17 & 22,22 \\
& Isoptères / Termitidae & 82 & 0,54 & 25 \\
& Lépidoptères / Hesperiidae & 18 & 0,11 & 33,33 \\
& Lépidoptères / Noctuidae & 20 & 0,13 & 38,88 \\
& Orthoptères / Acrididae & 121 & 0,79 & 69,44 \\
& Orthoptères / Gryllidae & 750 & 4,94 & 97,22 \\
\hline Badadon & Orthoptères / Tettigoniidae & 17 & 0,11 & 22,22 \\
& Coléoptères / Chrysomelidae & 56 & 0,39 & 63,88 \\
& Coléoptères / Curculionidae & 4 & 0,02 & 5,55
\end{tabular}




\begin{tabular}{|c|c|c|c|c|}
\hline & Coléoptères / Scarabaeidae & 96 & 0,63 & 47,22 \\
\hline & Homoptères / Cicadellidae & 268 & 1,76 & 77,77 \\
\hline & Homoptères / Derbidae & 14 & 0,09 & 16,66 \\
\hline & Isoptères / Termitidae & 70 & 0,46 & 33,33 \\
\hline & Lépidoptères / Cossoidae & 2 & 0,01 & 5,55 \\
\hline & Lépidoptères / Hesperiidae & 20 & 0,13 & 36,11 \\
\hline & Lépidoptères / Noctuidae & 18 & 0,11 & 25 \\
\hline & Orthoptères / Acrididae & 166 & 1,09 & 75 \\
\hline & Orthoptères / Gryllidae & 658 & 4,33 & 97,22 \\
\hline & Orthoptères / Tettigoniidae & 26 & 0,17 & 11,11 \\
\hline \multirow[t]{13}{*}{ Yaokro } & Coléoptères / Chrysomelidae & 81 & 0,53 & 69,44 \\
\hline & Coléoptères / Curculionidae & 7 & 0,04 & 8,33 \\
\hline & Coléoptères / Scarabaeidae & 528 & 3,48 & 83,33 \\
\hline & Homoptères / Cicadellidae & 512 & 3,37 & 100 \\
\hline & Homoptères / Delphacidae & 1 & 0,006 & 2,77 \\
\hline & Homoptères / Derbidae & 20 & 0,13 & 36,11 \\
\hline & Isoptères / Termitidae & 203 & 1,33 & 33,33 \\
\hline & Lépidoptères / Cossoidae & 3 & 0,01 & 2,77 \\
\hline & Lépidoptères / Hesperiidae & 12 & 0,07 & 19,44 \\
\hline & Lépidoptères / Noctuidae & 25 & 0,16 & 33,33 \\
\hline & Orthoptères / Acrididae & 176 & 1,16 & 86,11 \\
\hline & Orthoptères / Gryllidae & 1575 & 10,38 & 97,22 \\
\hline & Orthoptères / Tettigoniidae & 9 & 0,05 & 8,33 \\
\hline
\end{tabular}

Tableau 3: Ordres, familles, nombre d'insectes, taux de représentativité des prédateurs et parasitoïdes et constances des familles d'insectes recensés dans les différents sites d'étude à GrandLahou.

\begin{tabular}{llccc}
\hline Sites d'étude & Ordres / familles & $\begin{array}{c}\text { Nbre } \\
\text { d'insectes }\end{array}$ & $\begin{array}{c}\text { Taux de représentativité des } \\
\text { prédateurs et parasitoïdes } \\
(\%)\end{array}$ & $\begin{array}{c}\text { Constance } \\
(\%)\end{array}$ \\
\hline Adjadon & Coléoptères / Carabidae & 25 & 0,16 & 25 \\
& Coléoptères / Cicindelidae & 5 & 0,03 & 11,11 \\
& Coléoptères / Coccinellidae & 871 & 5,74 & 63,88 \\
& Coléoptères / Staphylinidae & 7 & 0,04 & 13,88 \\
& Dictyoptères / Mantidae & 2 & 0,01 & 5,55 \\
& Diptères / Asilidae & 10 & 0,06 & 16,66 \\
& Diptères / Conopidae & 76 & 0,50 & 41,66 \\
& Diptères / Dolichopodidae & 91 & 0,59 & 44,44 \\
& Diptères / Empididae & 1 & 0,006 & 2,77 \\
& Diptères / Pipunculidae & 4 & 0,02 & 11,11 \\
& Diptères / Sciomyzidae & 1 & 0,006 & 2,77 \\
& Diptères / Syrphidae & 19 & 0,12 & 22,22 \\
& Diptères / Tachnidae & 2 & 0,01 & 5,55 \\
& Hétéroptères / Reduviidae & 47 & 0,30 & 52,77 \\
Hyménoptères / Bethylidae & 249 & 1,64 & 88,88 \\
& Hyménoptères / Ceraphronidae & 2 & 0,01 & 5,55 \\
& Hyménoptères / Chalcididae & 165 & 1,08 & 72,22 \\
Hyménoptères / Crabronidae & 18 & 0,11 & 13,88
\end{tabular}


Hyménoptères / Diapriidae

Hyménoptères / Encyrtidae

Hyménoptères Evaniidae

Hyménoptères Figitidae

Hyménoptères / Formicidae

Hyménoptères / Ibaliidae

Hyménoptères /Ichneumonidae

Hyménoptères / Mutillidae

Hyménoptères / Pteromalidae

Hyménoptères / Scelionidae

Hyménoptères / Scoliidae

Hyménoptères / Sphecidae

Hyménoptères / Vespidae

Odonates / Coenagriidae

Thysanoptères / Thripidae

Coléoptères / Carabidae

Coléoptères / Cicindelidae

Coléoptères / Coccinellidae

Coléoptères / Staphylinidae

Dermaptères / Forficulidae

Dictyoptères / Mantidae

Diptères / Asilidae

Diptères / Conopidae

Diptères / Dolichopodidae

Diptères / Syrphidae

Diptères / Therevidae

Hétéroptères / Miridae

Hétéroptères / Reduviidae

Hyménoptères / Bethylidae

Hyménoptères / Braconidae

Hyménoptères / Chalcididae

Hyménoptères /Crabronidae

Hyménoptères /Encyrtidae

Hyménoptères / Evaniidae

Hyménoptères / Formicidae

Hyménoptères /Ichneumonidae

Hyménoptères / Platygastridae

Hyménoptères / Pteromalidae

Hyménoptères / Scelionidae

Hyménoptères / Scoliidae

\section{3}

14

18

4

6356

3

81

4

20

158

27

8

149

1

9

43

10

43

15

1

7

9

4

59

67

5

2

56

70

2

87

1

20

19

1978

29

11

30

120

3
0,01

0,09

0,11

0,02

41,89

0,01

0,53

0,02

0,13

1,04

0,17

0,05

0,98

0,006

0,05

0,55

0,12

0,55

0,19

0,01

0,09

0,11

0,05

0,76

0,86

0,06

0,02

0,72

0,90

0,02

1,12

0,01

0,25

0,24

25,54

0,37

0,14

0,38

1,54

0,03
11,11

27,77

36,11

5,55

100

2,77

55,55

5,55

13,88

80,55

16,66

13,88

77,77

2,77

8,33

33,33

11,11

30,55

16,66

2,77

5,55

16,66

5,55

33,33

55,55

5,55

2,77

41,66

55,55

5,55

47,22

2,77

19,44

25

100

27,77

2,77

22,22

55,55

5,55 





Tableau 4 : Indices de diversité de Shannon $(\mathrm{H})$ calculés pour chaque ordre d'insecte sur les sites à Grand-Lahou.

\begin{tabular}{|c|c|c|c|}
\hline Sites d'étude & Ordres & $\mathbf{H}$ & $\mathbf{E}$ \\
\hline \multirow[t]{13}{*}{ Adjadon } & Coléoptères & 1,419 & 0,466 \\
\hline & Collemboles & 0 & 0 \\
\hline & Dictyoptères & 0,132 & 0,191 \\
\hline & Diptères & 1,942 & 0,565 \\
\hline & Hétéroptères & 0,811 & 0,585 \\
\hline & Homoptères & 1,343 & 0,56 \\
\hline & Hyménoptères & 0,859 & 0,274 \\
\hline & Isoptères & 0 & 0 \\
\hline & Lépidoptères & 1,65 & 0,751 \\
\hline & Odonates & 0,693 & 1 \\
\hline & Orthoptères & 0,873 & 0,543 \\
\hline & Thysanoptères & 0 & 0 \\
\hline & Moyenne & $\mathbf{0 , 8 1}$ & 0,411 \\
\hline \multirow[t]{15}{*}{ Badadon } & Coléoptères & 1,82 & 0,642 \\
\hline & Collemboles & 0 & 0 \\
\hline & Dermaptères & 0 & 0 \\
\hline & Dictyoptères & 0,356 & 0,514 \\
\hline & Diptères & 2,6 & 0,789 \\
\hline & Hétéroptères & 0,627 & 0,570 \\
\hline & Homoptères & 1,14 & 0,518 \\
\hline & Hyménoptères & 1,223 & 0,408 \\
\hline & Isoptères & 0 & 0 \\
\hline & Lépidoptères & 1,366 & 0,848 \\
\hline & Neuroptères & 0,636 & 0,918 \\
\hline & Odonates & 0 & 0 \\
\hline & Orthoptères & 1,062 & 0,66 \\
\hline & Thysanoptères & 0 & 0 \\
\hline & Moyenne & 0,773 & 0,419 \\
\hline \multirow[t]{15}{*}{ Yaokro } & Coléoptères & 1,708 & 0,560 \\
\hline & Collemboles & 0 & 0 \\
\hline & Dictyoptères & 0,165 & 0,236 \\
\hline & Diptères & 2,453 & 0,744 \\
\hline & Embioptères & 0 & 0 \\
\hline & Hétéroptères & 0,772 & 0,479 \\
\hline & Homoptères & 1,165 & 0,485 \\
\hline & Hyménoptères & 0,695 & 0,225 \\
\hline & Isoptères & 0 & 0 \\
\hline & Lépidoptères & 1,25 & 0,776 \\
\hline & Neuroptères & 1,079 & 0,982 \\
\hline & Odonates & 0,636 & 0,918 \\
\hline & Orthoptères & 0,629 & 0,391 \\
\hline & Thysanoptères & 0 & 0 \\
\hline & Moyenne & 0,708 & 0,414 \\
\hline
\end{tabular}




\section{DISCUSSION}

Ces travaux effectués nous ont permis de collecter une population importante mais probablement non exhaustive d'insectes dans les cocoteraies infectées par la maladie du jaunissement mortel du cocotier à GrandLahou. La plupart de ces insectes ont fait l'objet d'identification.

Le site de Yaokro est celui sur lequel plus d'insectes ont été collectés. Cela pourrait s'expliquer par le fait qu'étant orienté vers le continent, les espèces végétales sur ce site sont un peu plus diversifiées que les autres sites d'étude et attireraient divers insectes. Sur ce site, plusieurs plantes autres que le cocotier (le cacaoyer, l'avocatier et autres) se cultivent. Il est probable que la diversité des planteshôtes, la biomasse et le jeu des interactions biotiques se combinent pour définir le niveau d'abondance dans les différents milieux. Parmi les interactions biotiques importantes, on peut citer la compétition et la prédation (Speight et al., 2008).

Des deux autres sites, le site d'Adjadon est celui sur lequel a été récolté plus d'insectes. Cela pourrait s'expliquer par le fait que ce site, abritant des jeunes cocotiers de moins de 10 ans, est plus apprécié par les ravageurs. La plupart des insectes ravageurs de cocotier s'attaquent aux plants très jeunes à cause de la tendresse des différents organes (Beugré et al., 2017). Par contre, le site de Badadon est celui où le moins d'insectes a été collecté par rapport aux autres. Ceci serait dû à l'âge des plants qui s'y trouvent et aussi au sol qui étant sableux, ne favorisant pas le développement d'une diversité d'espèces végétales. Mariau (2000) a affirmé que l'importance de l'entomofaune est elle-même liée à la biodiversité végétale et à l'âge des palmiers. Ainsi, les caractéristiques propres à chaque habitat, en termes de composition de la végétation, peuvent induire des différences, quantitatives et probablement qualitatives, dans l'entomofaune présente. Les conditions édaphiques (porosité, texture et structure, imperméabilité) conditionnent la composition (diversité spécifique) de la végétation de chaque type d'habitat. Ces conditions influencent la distribution locale des insectes associés aux cortèges de plantes-hôtes (Lamarre, 2013).

Pour évaluer l'impact des insectes étudiés sur leur milieu, ceux-ci ont été classifiés par groupe biologique. Les constances des insectes ravageurs des différents sites d'étude ont révélé qu'il existe des familles constantes, accessoires, accidentelles et très accidentelles à Adjadon. La présence des familles déprédatrices constantes sur ce site s'expliquerait par le fait de la présence des jeunes plants de cocotiers (moins de 10 ans). Selon Mariau (2000), il a été constaté que les pullulations de ravageurs sont beaucoup plus fréquents dans les jeunes palmeraies c'est-à-dire au cours des dix premières années de culture.

La présence des familles déprédatrices constantes à Badadon pourrait s'expliquer par le fait que les parcelles de cocotiers, étant des plantations villageoises, sont mal entretenues. Les mauvaises herbes qui sont des sources d'infestation ne sont souvent pas enlevées.

En ce qui concerne le site de Yaokro, la présence de plusieurs familles déprédatrices constantes a été observée. La présence de familles constantes serait dû au fait que la plupart des cocoteraies de Yaokro sont fortement détruites à cause de la maladie du jaunissement mortel du cocotier et ces cocoteraies sont souvent remplacées par des plantations de manioc, de cacao ou autres cultures. Ce qui pourrait augmenter la population de ces familles déprédatrices constantes. On peut aussi signaler que la nature des espèces végétales les plus abondantes dans chaque habitat est un autre facteur qui pourrait expliquer les variations d'abondance par parcelle. Les habitats seminaturels (forêts, bordures de champ, bandes enherbées, jachères) sont les zones sources d'un certain nombre d'insectes ravageurs (Marshall, 2004).

Pour ce qui est des familles prédatrices et parasitoïdes recensées dans les différentes parcelles de ces sites d'études, toutes les catégories de familles d'insectes ont été retrouvées sur le site d'Adjadon. Les Hyménoptères, particulièrement les guêpes, sont des parasitoïdes ou prédatrices des œufs 
des Hémiptères Cicadomorphes et des chenilles des Lépidoptères foreurs de tiges. La présence de toutes ces familles prédatrices et parasitoïdes pourrait se justifier par le fait qu'elles peuvent être divisées en prédateurs généralistes et en prédateurs spécialistes. Un prédateur généraliste présente l'avantage de ne pas être dépendant d'une proie spécifique et en absence de celle-ci, il peut consommer une autre et survivre. Un prédateur spécialisé présente lui l'avantage de ne pas être distrait par une proie alternative (Symondson et al., 2002). En contrepartie, la présence de cette proie est nécessaire à son arrivée et à son développement, et la disparition de celle-ci implique sa mort ou sa migration. La présence d'un prédateur généraliste est donc plus facile à pérenniser (Hazem, 2010).

Tout comme à Adjadon, des familles prédatrices et parasitoïdes de toutes catégories ont été collectées à Badadon. La présence de ces familles constantes serait relative à celle des déprédateurs à cause de l'enherbement constaté dans certaines parties du champ. On admet communément que les habitats seminaturels sont les zones sources d'insectes auxiliaires mais aussi d'un certain nombre d'insectes ravageurs (Denys et Tscharntke, 2002 ; Marshall, 2004). D'ailleurs, Bianchi et al. (2006) ont montré que la pression des insectes ravageurs, toutes cultures confondues, était réduite dans $45 \%$ des situations où le paysage était qualifié de complexe alors que dans $75 \%$ de ces situations, où le paysage était complexe, le nombre et l'activité des ennemis naturels étaient augmentés.

Des familles prédatrices et parasitoïdes d'insectes ravageurs de cocotier ont été recensées à Yaokro. Ces familles ont été regroupées en familles constantes, accessoires, accidentelles et très accidentelles. La présence de ces familles serait due à la présence d'autres cultures autres que le cocotier sur ce site. Des plantes de couverture, plantes compagnes ou associations d'espèces permettent d'augmenter la biodiversité dans les champs cultivés, ce qui permettrait d'augmenter les ressources pour les insectes auxiliaires généralistes et de perturber le milieu pour les insectes ravageurs. Ferron et Deguine (2005) et Broad et al. (2008) ont montré une réduction significative des insectes nuisibles dans les systèmes de cultures associées, comparativement aux monocultures de mêmes espèces.

Les indices de diversité de Shannon calculés pour les différents sites d'étude ont montré que ces indices varient de 0,708 à 0,81 bits. Ces indices de diversité sont surtout faibles, cela se justifierait par la faible diversité du couvert végétal qui est presque le même dans tous sites d'études. Selon (Lewinsohn et Roslin, 2008), la diversité des insectes peut être expliquée, en partie, par une plus grande diversité de plantes. Les valeurs de l'indice d'équitabilité, pour les trois sites d'étude sont également inférieures à 50\%. Ce qui dénote d'un peuplement en fort déséquilibre, d'où un déséquilibre dans la distribution taxonomique. Les habitats à fortes ressources disponibles supportent des communautés d'insectes plus diverse et abondante que les habitats à faible ressources (Lamarre, 2013).

\section{Conclusion}

Cette étude a permis de recenser 39 514 insectes dont 15171 insectes à Adjadon, 7743 à Badadon et 16600 insectes à Yaokro. L'étude de la constance des ravageurs de cocotier a révélé des familles constantes, accessoires, accidentelles et très accidentelles au niveau des différentes parcelles des sites d'étude. Tout comme la constance des ravageurs, l'étude de la constance des prédateurs et parasitoïdes a révélé pour les parcelles des différents sites, des familles constantes, accessoires, accidentelles et très accidentelles.

Les indices de diversité de Shannon calculés pour les parcelles sont faibles et montrent que les ordres d'insectes identifiés sont moins diversifiés. Les indices d'équitabilité étudiés confirment également que l'abondance des familles répertoriées est très déséquilibrée au sein des ordres identifiés. Ce travail a permis de montrer qu'à côté des ravageurs cohabitent les insectes utiles (prédateurs et parasitoïdes). Notre suggestion 
à l'endroit des producteurs de cocotier porte sur l'utilisation de façon raisonnée les pesticides afin de maintenir les populations d'insectes utiles dans les plantations.

\section{CONFLIT D'INTERETS}

Les auteurs déclarent n'avoir aucun conflit d'intérêts concernant cet article.

\section{CONTRIBUTIONS DES AUTEURS}

NIB, initiatrice de l'article, a saisi les données de l'inventaire, a procédé à l'analyse des données, à leur interprétation et à la rédaction du document. KEK, KDK, ASDD, KKSL et WAMPD ont contribué à la révision des données saisies et analysées, et à la révision critique du contenu de l'article. KA a contribué à l'interprétation des données et à la révision critique du contenu de l'article. DM a contribué à la l'analyse des données et leur interprétation, a orienté, lu et corrigé le document.

\section{REFERENCES}

Allou K. 2009. Etude comportementale et moyens de lutte par piégeage olfactif et par répulsion contre Oryctes monoceros Olivier (Coleoptera, Dynastidae), ravageur du cocotier (Cocos nucifera $\mathrm{L}$.,) en basse Côte d'Ivoire. Thèse d'Etat èsSciences Naturelles, UFR Biosciences, Université de Cocody, Abidjan, Côte d'Ivoire, p. 169.

Amenan AY, Atcham TA, Pohe J, D'Almeida AM, Zana P. 2012. Association d'organismes de type mycoplasmes avec le dépérissement mortel des cocotiers de Grand-Lahou en Côte d'Ivoire. Int. J. Biol. Chem. Sci., 6(3): 959-984. DOI : http://ajol.info/index.php/ijbcs

Assa R, Konan JL, Prades A, Nemlin J, Koffi E. 2006. Physicochemical characteristics of kernel during fruit maturation of four coconut cultivars (Cocos nucifera L.). African Journal of Biotechnology, 9(14): 2136-2144.

DOI: http://www.academicjournals.org/AJB

Beugre NI, Yao SDM, Allou K, Dagnogo M. 2017. Diversité de la faune d'insectes associée à la culture du cocotier à Port-
Bouët, Côte d'Ivoire. African Crop Science Journal, 25(2): 157-175. DOI : http://dx.doi.org/10.4314/acsj.v25i2.3

Bianchi FJJA, Booij CJH, Tscharntke T. 2006. Sustainable pest regulation in agricultural landscapes: a review on landscape composition, biodiversity and natural pest control. Proc Biol Sci., 273(1595): $\quad$ 1715-1727. DOI: 10.1098/rspb.2006.3530

Bigot L, Bodot P. 1973. Contribution à l'étude biocénotique de la garrigue à Quercus coccifera - Composition biotique du peuplement des invertébrés. Vie et Vol. Milieu, 23(2): 229-249.

Broad ST, Schellhorm NA, Lisson SN, Mendham NJ, Corkrey R. 2008. Host location and parasitism of Brevicoryne brassicae in diversified broccoli cropping systems. Entomol Exp Appl., 129(2): $166-171 . \quad$ DOI : https://doi.org/10.1111/j.15707458.2008.00762.x.

CIRAD. 2014. Les jaunissements mortels du cocotier. Vers une stratégie de lutte intégrée. Centre de Coopération Internationale en Recherche Agronomique pour le développement (CIRAD) : Montpellier.

CNRA. 2006. Bien cultiver le cocotier en Côte d'Ivoire. CNRA, p. 4.

CNRA. 2012. Diagnostic et gestion de la maladie provoquant la mortalité du cocotier dans le département de GrandLahou. Fiche cocotier n ${ }^{\circ} 6$, p. 4.

CNRA. 2013. Le CNRA en 2013. Abidjan, CNRA, p. 54.

CNRA. 2014. Economie (Abidjan.net). Centre National de Recherche Agronomique. Activités de recherche: Grand-Lahou : la maladie du jaunissement mortel gagne du terrain.

Dajoz R. 1982. Précis d'écologie. Ecologie fondamentale et appliquée. Ed. GauthierVillars : Paris.

Dajoz R. 2006. Précis d'Ecologie (8e Edn). Dunod : Paris.

Delvare G, Aberlenc HP. 1989. Les insectes d'Afrique et d'Amérique Tropicale; Clés pour la Reconnaissance des Familles. 
Prifas-Cirad: 34032 Montpellier Cedex 1, France.

Denys C, Tscharntke T. 2002. Plant-insect communities and predator-prey ratios in field margin strips, adjacent crop fields, and fallows. Oecologia, 130(2): 315324.

DOI: https://doi.org/10.1007/s004420100796

FAO Oil World. 2010. Perspective de l'alimentation. Evaluation des marchés. Département économiques et sociales.(http://www.Fao.org. Html ; 18/11/2011):36-107.

Ferron P, Deguine JP. 2005. Crop protection, biological control, habitat management and integrated farming, a review. Agron Sustain Dev., 25(1): 17-24. DOI: http://dx.doi.org/10.1051/agro:2004050.

Gunn BF, Baudouin L, Olsen KM. 2011. Independent origins of cultivated coconut (Coconut nucifera L.) in the old world tropics. PLoS One, 6(6): e21143. DOI : 10.1371 /journal.pone. 0021143

Hazem D. 2010. Rôle des ennemis naturels dans la lutte biologique contre le puceron cendré, Dysaphis plantaginea Passerini (Hemiptera aphididae) en vergers de pommiers. Thèse de doctorat, Sciences agricoles, Université d'Avignon, p. 253.

Lamarre GPA. 2013. Rôle des insectes phytophages dans la diversité des arbres des forêts tropicales humides. Thèse de Doctorat, Université des Antilles et de la Guyane. Faculté des Sciences exactes et naturelles, p. 205.

Lewinsohn T M, Roslin T. 2008. Four ways towards tropical herbivore megadiversity. Ecol Lett., 11(4): 398416. DOI: $\quad 10.1111 /$ j.14610248.2008.01155.x. Epub2008 Jan 31.

Mariau D. 2000. Problèmes entomologiques en replantation des palmeraies et des cocoteraies. Oléagineux, Corps, Gras, Lipides, 7(2): 203-206.

Marshall EJP. 2004. Agricultural landscapes : Field margin habitats and their interaction with crop production. Journal of Crop Improvement, 12(1): 365-404. DOI: 10.1300/j411v12n01_05

Konan JL, Allou K, Lekadou TT, Tahouo O. 2013. Etude de la maladie du cocotier identifiée dans le département de GrandLahou. Projet FIRCA/Cocotier CNRA COC NO 588. Rapport final, Mai 2012 Septembre 2013, p. 34.

ProMED. 2013. Undiagnosed disease, coconut palm-Côte d'Ivoire. ProMED mail post 20130301.1563457. International Society of Infectious Disease.

Speight MR, Hunter MD, Watt AD. 2008. Ecology of Insect: Concepts and applications. $2^{\text {nd }}$ Edition: WileyBlackwell.

Roth M. 1980. Initiation à la morphologie, la systématique et la biologie des insectes. Office de la Recherche Scientifique et Technique Outre-Mer (ORSTOM) : Bondy.

Symondson WOC, Sunderland KD, Greenstone MH. 2002. Can generalist predators be effective biocontrol agents? Annu Rev of Entomol., 47: 561-594. DOI:

https://doi.org/10.1146/annurev.ento.47. 091201.145240

Van Der Vossen HAM, Chipungahelo GSE. 2007. Cocos nucifera L. In Végétable oils/Oléagineux [CD-ROM], Van der Vossen HAM, Mkamilo GS (Eds). Fondation PROTA: Wageningen, PaysBas / Backbuys Publishers: Eeiden, Pays-Bas; 1-17. 